\title{
diproperm: An R Package for the DiProPerm Test
}

\author{
by Andrew G. Allmon, J.S. Marron, and Michael G. Hudgens
}

\begin{abstract}
High-dimensional low sample size (HDLSS) data sets frequently emerge in many biomedical applications. The direction-projection-permutation (DiProPerm) test is a two-sample hypothesis test for comparing two high-dimensional distributions. The DiProPerm test is exact, i.e., the type I error is guaranteed to be controlled at the nominal level for any sample size, and thus is applicable in the HDLSS setting. This paper discusses the key components of the DiProPerm test, introduces the diproperm $\mathrm{R}$ package, and demonstrates the package on a real-world data set.
\end{abstract}

\section{Introduction}

Advancements in modern technology and computer software have dramatically increased the demand and feasibility to collect high-dimensional data sets. Such data introduce analytical challenges which require the creation of new and adaptation of existing statistical methods. One such challenge is that we may observe many more covariates (features) $p$ than the number of observations $N$, especially in small sample size studies. These data structures are known as high-dimensional, low sample size (HDLSS) data sets, or "small $N$, big $p . "$

HDLSS data frequently occur in many health-related fields. For example, in genomic studies, a single microarray experiment can produce tens of thousands of gene expressions compared to the few samples studied, often being less than a hundred (Alag, 2019). In medical imaging studies, a single region of interest for analysis in an MRI or CT-scan image often contains thousands of features compared to the small number of samples studied (Limkin et al., 2017). In the pre-clinical evaluation of vaccines and other experimental therapeutic agents, the number of biomarkers measured (e.g., immune responses) may be much greater than the number of samples studied (e.g., mice, rabbits, or non-human primates) (Kimball et al., 2018).

One common task in the HDLSS setting entails comparing the distribution of covariates between two groups (classes). For example, in pre-clinical studies of vaccines, investigators may wish to compare the distribution of biomarkers between animals who survive to a certain time point and animals who do not survive. In the example presented below, the distribution of $p=112$ covariates is compared between edible and poisonous mushrooms. The covariate distributions may be compared between two samples using the direction-projection-permutation (DiProPerm) test (Wei et al., 2016). This test is well-suited for the HDLSS setting because the test is exact, i.e., the type I error is guaranteed to be controlled at the nominal level for any sample size. Below we discuss the key components of the DiProPerm test, introduce the diproperm $\mathrm{R}$ package, and demonstrate the use of the package on a real-world data set.

\section{DiProPerm Test}

To compare the distribution of covariates between two groups, the DiProPerm tests use one-dimensional projections of the data based on a binary linear classifier to construct a univariate test statistic and then permutes class labels to determine the sampling distribution of the test statistic under the null. The details of the DiProPerm test are as follows.

Let $X_{1}, \ldots, X_{n} \sim F_{1}$ and $Y_{1}, \ldots, Y_{m} \sim F_{2}$ be independent random samples of $p$ dimensional random vectors from multivariate distributions $F_{1}$ and $F_{2}$ where $p$ may be larger than $m$ and $n$. The DiProPerm test evaluates the hypotheses

$$
H_{0}: F_{1}=F_{2} \text { versus } H_{1}: F_{1} \neq F_{2}
$$

The test entails three steps:

1. Direction: find the normal vector to the separating hyperplane between two samples after training a binary linear classifier

2. Projection: project data on to the normal vector and calculate a univariate two-sample statistic

3. Permutation: conduct a permutation test using the univariate statistic as follows:

(a) Permute class membership after pooling samples 
(b) Re-train binary classifier and find the normal vector to the separating hyperplane

(c) Recalculate the univariate two sample statistic

(d) Repeat a-c multiple times (say 1000) to determine the sampling distribution of the test statistic under the null $H_{0}$

(e) Compute $p$-value by comparing the observed statistic to the sampling distribution

Different binary linear classifiers may be used in the first step of DiProPerm. Linear discriminant analysis (LDA), particularly after conducting principal component analysis (PCA), is one possible classifier for the direction step. However, using LDA with PCA in the HDLSS setting has some disadvantages, including lack of interpretability, sensitivity to outliers, and tendency to find spurious linear combinations due to a phenomenon known as data piling (Aoshima et al., 2018; Marron et al., 2007). Data piling occurs if data are projected onto some projection direction, and many of the projections are the same or piled on one another. The support vector machine (SVM) is another popular classifier (Hastie et al., 2001). The SVM finds the hyperplane that maximizes the minimum distance between data points and the separating hyperplane. However, the SVM can also suffer from data piling in the HDLSS setting. To overcome data piling, the distance weighted discrimination (DWD) classifier was developed (Marron et al., 2007). The DWD classifier finds the separating hyperplane minimizing the average inverse distance between data points and the hyperplane. The DWD performs well in HDLSS settings with good separation and is more robust to data piling.

In the second step of DiProPerm, a univariate statistic is calculated using the projected values onto the normal vector to the separating hyperplane from the first step. Suppose $x_{1}, \ldots, x_{n}$ and $y_{1}, \ldots, y_{m}$ are the projected values from samples $X_{1}, \ldots, X_{n}$ and $Y_{1}, \ldots, Y_{m}$, respectively. One common choice for the univariate test statistic for DiProPerm includes the difference of means statistic, $|\bar{x}-\bar{y}|$. Other two-sample univariate statistics such as the two-sample t-statistic or difference in medians are also possible for use with the DiProPerm.

The last step of DiProPerm entails determining the distribution of the test statistic under the null. In this step, the two samples are pooled, class labels are permuted, then a univariate statistic is calculated. Repeat this process multiple times (say 1000) to determine the sampling distribution of the test statistic under the null $H_{0}$. P-values are then calculated by the proportion of statistics higher than the original value.

When the DiProPerm test is implemented using the DWD classifier, it is common practice to look at the loadings of the DWD classifier (An et al., 2016; Nelson et al., 2019). The DWD loadings represent the relative contribution of each variable to the class difference. A higher absolute value of a variable's loading indicates a greater contribution for that variable to the class difference. The loadings vector is a unit vector; thus, the individual loadings range from -1 to 1 , and the sum of the squares of the loadings equals one. The loadings direction vector points from the negative to positive class. Thus, positive entries correspond to variables that tend to be larger for the positive class. Combining the use of the DiProPerm and evaluation of the DWD loadings in applications can provide insights into high-dimensional data and be used to generate rational hypotheses for future research.

The DiProPerm test has been used in several areas of biomedical research, including osteoarthritis and neuroscience (An et al., 2016; Bendich et al., 2016; Nelson et al., 2019). However, currently, there does not exist an R package that implements DiProPerm. Therefore we developed diproperm, a free, publicly available $\mathrm{R}$ software package to analyze data from two high-dimensional distributions (Allmon et al., 2020). Functions in the diproperm package allow users to conduct the DiProPerm test and create corresponding diagnostic plots. Loadings for the binary linear classifier are also available for display in order from highest to lowest relative to their contribution toward the separation of the two distributions.

\section{Package Overview}

The diproperm package is comprised of three functions:

- DiProPerm(): Conducts the DiProPerm test

- $\operatorname{plotdpp():~Plots~diagnostics~from~the~DiProPerm~test~}$

- loadings(): Returns the variable indices with the highest loadings in the binary classification. The absolute values of the loading values indicate a variable's relative contribution toward the separation between the two classes. The loadings vector is a unit vector, thus, the sum of its squares must be equal to one and range from -1 to 1 . Also, the loadings direction vector points from the negative to positive class. Thus, positive entries correspond to variables that are larger for the positive class. 
The diproperm package can be installed from CRAN using the code in the 'Example' section below. Additionally, the development version of the package can be installed from GitHub ("allmondrew/diproperm").

\section{Example}

The example below creates a Gaussian data set containing 100 samples, 2 features, clustered around 2 centers with a standard deviation of 2 . The class labels are then re-classified to -1 and 1 to match the input requirements of DiProPerm(). The DiProPerm test is then conducted using the DWD classifier, the mean difference univariate statistic, and 1000 permutations. The results from DiProPerm() are then displayed with plotdpp(). Last, the top five indices of the highest absolute loadings are listed.

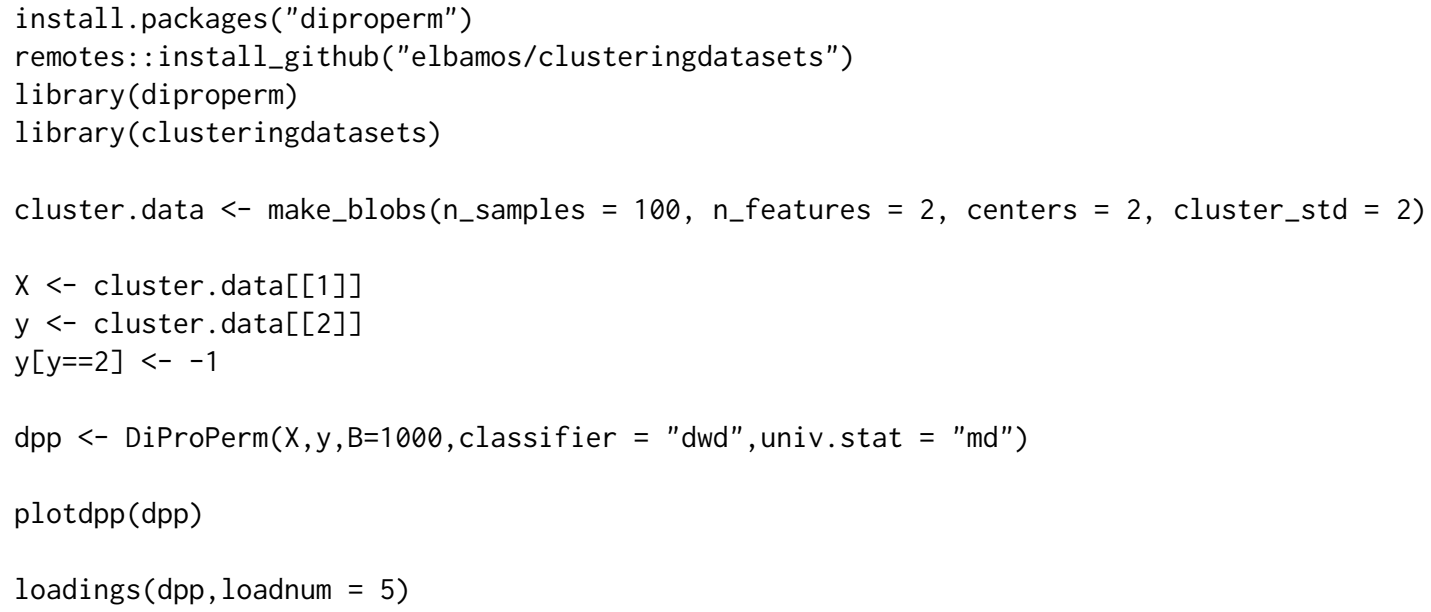

\section{Description}

The main function to be called first by the user is DiProPerm(), which takes in an $n \times p$ data matrix and a vector of $n$ binary class labels both provided by the user. Factor variables for the data matrix must be coded as $0 / 1$ dummy variables, and the class labels for the vector of binary class labels must be coded as -1 and 1. By default, the DiProPerm() uses the DWD classifier, the mean difference as the univariate statistics, and 1000 balanced permutations. The permutations are balanced in the sense that after relabeling, the new -1 group contains $n / 2$ members from the original -1 group and $n / 2$ members not from the original -1 group. Users can also implement an unbalanced, randomized permutation design if desired. DiProPerm() implements DWD from the genDWD function in the DWDLargeR package (Lam et al., 2018a,b). The penalty parameter, C, in the genDWD function is calculated using the penaltyParameter function in DWDLargeR. DWDLarge R has several parameters which are set to recommended default values. More details on the algorithm used to compute genDWD and penal tyParameter can be found in Lam et al. (2018b). Other options included in DiProPerm() for the binary linear classifier are the mean difference direction " $m d^{\prime \prime}$ and support vector machine "svm". DiProPerm() uses parallel processing to delegate computation to the number of cores on the user's computer for increased efficiency. DiProPerm() returns a list of the observed data matrix, vector of observed class labels, observed test statistic, projection scores used to compute the observed test statistic, the loadings of the binary classification, the z-score, cutoff value for an $\alpha$ level of significance, the $p$-value for the DiProPerm test, a list of each permutation's projection scores and permuted class labels, and a vector of permuted test statistics the size of the number of permutations used.

After calling DiProPerm(), the function $\operatorname{plotdpp()}$ can be used to create a panel plot for assessing the diagnostics of the DiProPerm test. plotdpp() takes in a DiProPerm list and the user may specify which diagnostics they would like to display. By default, plotdpp() displays a facet plot with the observed score distribution, the projection score distribution of the permutation with the smallest test statistic value, the projection score distribution of the permutation with the largest test statistic value, and the test statistic permutation distribution. For the permutation distribution plot, the zscore, cutoff value, observed test statistic and $p$-value are displayed on the graph. Larger individual graphs may be displayed by using the plots option in $\operatorname{plotdpp().~Additional~graphs~include~the~}$ projection score distributions for the first and second permutations. The diagnostic plots show the user the characteristics of their data and facilitate the visual assessment of the separation of the two high-dimensional distributions being tested.

Lastly, after calling the DiProPerm(), the user may call the loadings() function. The loadings() function returns the variable indices in the data matrix which have the highest absolute loadings in the 
binary classification. Because the loadings vector is a unit vector, the sum of the squares of loadings is constrained to equal to one, with each loading between -1 to 1 . The loadings direction vector points from the negative to the positive class. Thus, positive entries correspond to variables that tend to be larger for the positive class. Higher absolute loading values indicate a greater contribution for a particular variable toward the separation between the two classes. By default, loadings() returns the indices for all variables sorted by their absolute loading value. Therefore, the top variable index is the variable which contributes the most toward the separation of the two classes, and the last variable is the one which contributes the least. The user may also change the number of loadings displayed.

\section{Application}

To illustrate the use of the diproperm package, consider the mushrooms data set, which is freely available from the UCI Machine Learning Repository (Dua and Graff, 2019) and within diproperm. This data set includes various characterizations of 23 species of gilled mushrooms in the Agaricus and Lepiota families. Each mushroom species is labeled as either definitely edible or poisonous/unknown. There are $n=8124$ mushrooms in total, and $p=112$ binary covariates coded as $0 / 1$ corresponding to 22 categorical attributes. Below, we demonstrate the diproperm package functionality using data from the first $n=50$ mushrooms in the data set.

\section{Step 1: Load and clean the data}

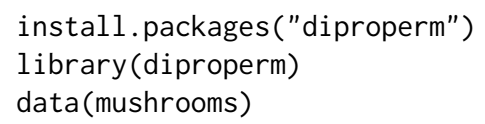

The above code installs the diproperm package and loads the mushroom data into R. Now, let us check the structure of the data to make sure it is compatible with DiProPerm().

$\operatorname{dim}($ mushrooms $\$ \mathrm{X}$ )

[1] 1128124

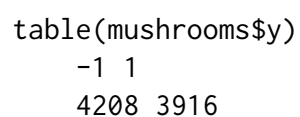

The vector of class labels must be -1 or 1 for DiProPerm(), which is the case for this data. However, the data set is in $p \times n$ format. For DiProPerm(), the dataset must be in $n \times p$ format. This can be done using the transpose function from the Matrix package in R (Bates and Maechler, 2019). After taking the transpose, we subset the data and vector of class labels to the first 50 observations and store the results.

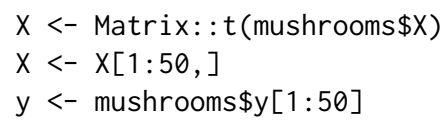

\section{Step 2: Conduct DiProPerm}

Now, that the data is in the proper format the call to DiProPerm() is as follows:

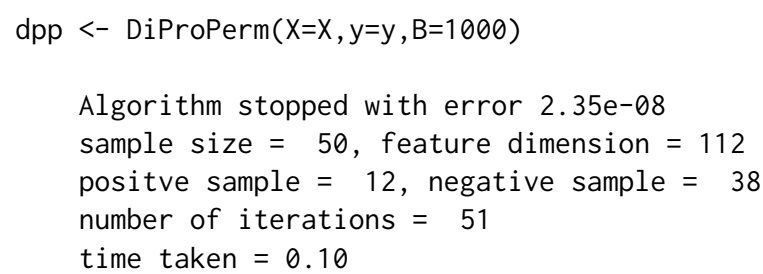

Characteristics of the DWD algorithm used to find the solution for the observed data are displayed by DiProPerm(). The algorithm took 51 iterations and 0.10 seconds to converge to the tolerance threshold with a zero percent classification error on the training data set. The runtime for 1000 permutations was less than 3 minutes on a four-core machine but would be faster on a machine with more cores. The dpp object stores the output list from DiProPerm() described in the package. Storing the information allows us to plot the diagnostics in the next step. 


\section{Step 3: Plot diagnostics}

$\operatorname{plotdpp(dpp)}$
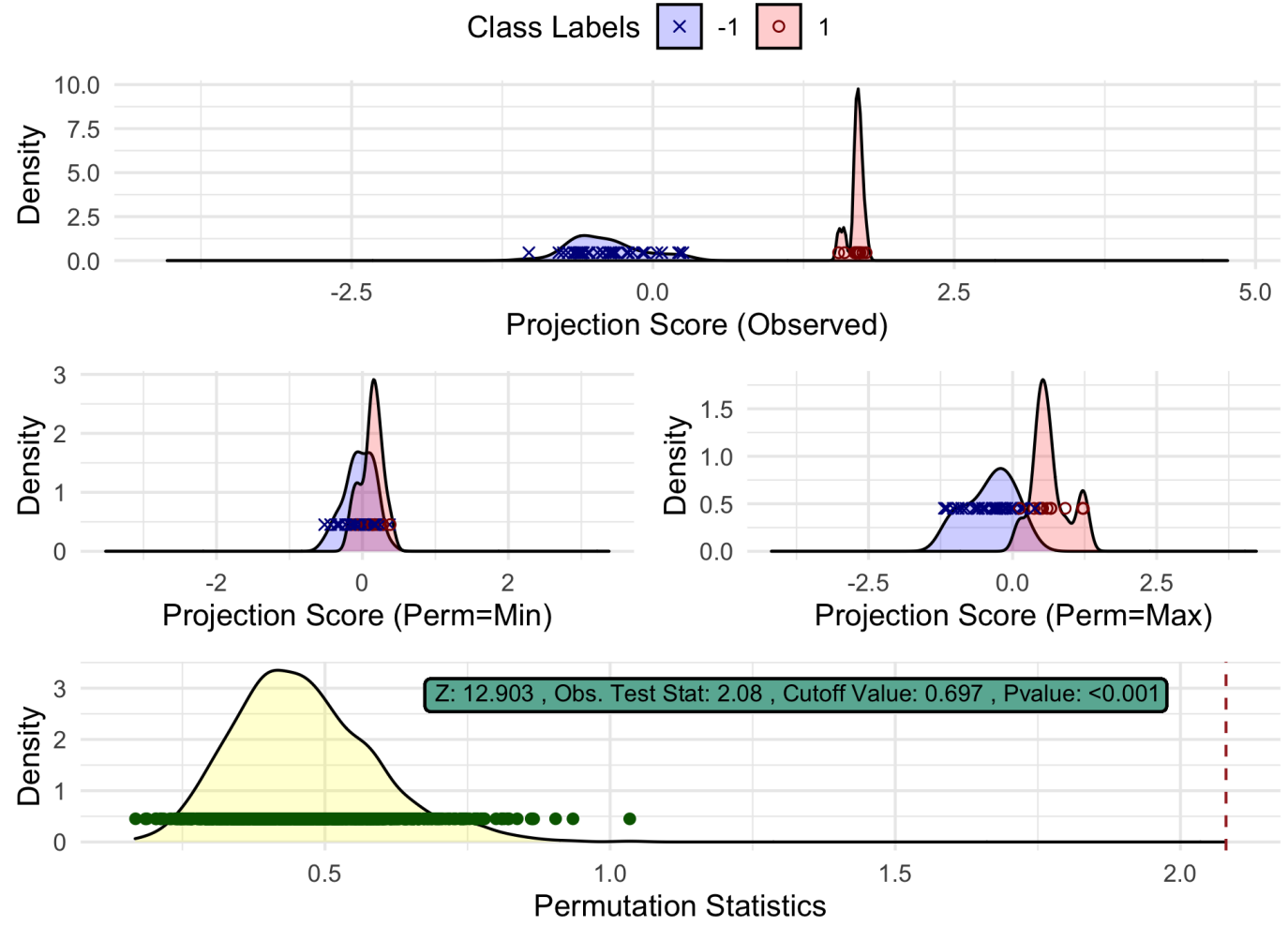

Figure 1: The diagnostic plot from $\operatorname{plotdpp}()$ for the mushrooms data set. The top graph is the observed projection score distribution of the two classes, the two middle graphs are the projection score distributions of the permutation with the smallest and largest test statistic value, and the bottom graph is the test statistic permutation distribution with the observed statistic value marked by the red dotted line.

Figure 1 displays the default diagnostics for a DiProPerm list. From the observed projection score distribution, one can see clear separation between the two classes. Also, from the projected score distributions of the permutations, which yield the smallest and largest test statistic, we see the score distributions overlap well, so there is some visual justification that the distributions in the observed plot are truly different. Lastly, the bottom plot shows the sampling distribution under the null is located around 0.4 while the observed test statistic is greater than 2. Each individual plot can also be output by the following set of commands:

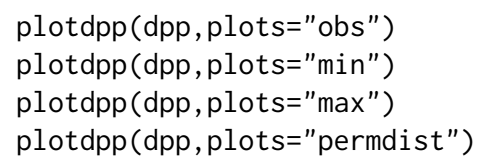

The permutation $p$-value in Figure 1 suggests that the two high-dimensional distributions of mushroom attributes are indeed different between the two classes. Also displayed is a z-score, calculated by fitting a Gaussian distribution to the test statistic permutation distribution. The mushroom data z-score 12.9 indicates the observed test statistic is approximately 13 standard deviations from the expected value of the test statistic under the null. Finally, the cutoff value 0.697 is displayed, corresponding to the critical value for a hypothesis test at the 0.05 significance level.

\section{Step 4: Examine loadings}

In order to assess which variables contributed most toward the separation in step 3, we can print the top five contributors with the code 


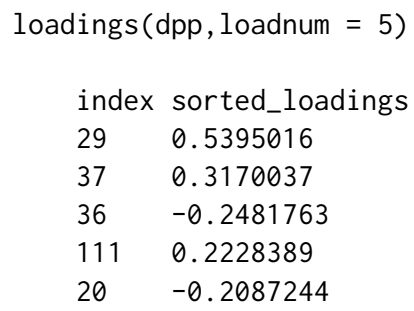

The top five contributors toward the separation seen in the observed distribution in Figure 1 are indices 29, 37, 36, 111, and 20. These indices correspond to a pungent odor, narrow gill size, broad gill size, urban habitat, and yellow cap color, respectively. For these data, $y=1$ corresponds to poisonous and $y=-1$ to edible; thus, loadings with positive entries, such as pungent odor, are indicative of poisonous mushrooms. These results are similar to previous analyses, which have also found odor, gill size, habitat, and cap color predictive of mushroom edibility (Pinky et al., 2019; Wibowo et al., 2018).

\section{Summary}

DiProPerm is an exact test for comparing two high-dimensional distributions. The diproperm package allows the user to visually assess and conduct a DiProPerm test to determine if there is a difference between the high-dimensional distributions of two classes and, if so, evaluate the key features contributing to the separation between the classes.

\section{Acknowledgments}

This work was supported by the National Institute of Allergy and Infectious Diseases (NIAID) of the National Institutes of Health (NIH) under award number R37AI054165. The content is solely the responsibility of the authors and does not necessarily represent the official views of the NIH. The authors thank two anonymous reviewers for helpful comments.

\section{Bibliography}

A. Alag. Machine learning approach yields epigenetic biomarkers of food allergy: A novel 13gene signature to diagnose clinical reactivity. PLOS ONE, 14(6):e0218253, 2019. URL https: //doi.org/10.1371/journal. pone.0218253. [p266]

A. G. Allmon, J. Marron, and M. G. Hudgens. diproperm: Conduct Direction-Projection-Permutation Tests and Display Plots, 2020. URL https://CRAN. R-project.org/package=diproperm. R package version 0.1.0. [p267]

H. An, J. S. Marron, T. A. Schwartz, J. B. Renner, F. Liu, J. A. Lynch, N. E. Lane, J. M. Jordan, and A. E. Nelson. Novel statistical methodology reveals that hip shape is associated with incident radiographic hip osteoarthritis among African American women. Osteoarthritis and Cartilage, 24 (4):640-646, 2016. doi: 10.1016/j.joca.2015.11.013. URL https://www.ncbi.nlm.nih.gov/pubmed/ 26620089https://www.ncbi.nlm.nih.gov/pmc/articles/PMC4799754/. [p267]

M. Aoshima, D. Shen, H. Shen, K. Yata, Y.-H. Zhou, and J. S. Marron. A survey of high dimension low sample size asymptotics. Australian E New Zealand Journal of Statistics, 60(1):4-19, 2018. doi: 10.1111/anzs.12212. URL https://doi.org/10.1111/anzs. 12212. [p267]

D. Bates and M. Maechler. Matrix: Sparse and Dense Matrix Classes and Methods, 2019. URL https: //CRAN.R-project. org/package=Matrix. R package version 1.2-18. [p269]

P. Bendich, J. S. Marron, E. Miller, A. Pieloch, and S. Skwerer. Persistent Homology Analysis of Brain Artery Trees. The Annals of Applied Statistics, 10(1):198-218, 2016. doi: 10.1214/15AOAS886. URL https://www.ncbi.nlm.nih.gov/pubmed/27642379https://www.ncbi.nlm.nih. gov/pmc/articles/PMC5026243/. [p267]

D. Dua and C. Graff. UCI Machine Learning Repository, 2019. URL https://archive.ics. uci .edu/ml/ datasets/Mushroom. [p269] 
T. Hastie, R. Tibshirani, and J. H. Friedman. The Elements of Statistical Learning : Data Mining, Inference, and Prediction. New York: Springer, 2001. URL https://catalog. lib.unc.edu/catalog/ UNCb4019902. [p267]

A. K. Kimball, L. M. Oko, B. L. Bullock, R. A. Nemenoff, L. F. van Dyk, and E. T. Clambey. A Beginner's Guide to Analyzing and Visualizing Mass Cytometry Data. The Journal of Immunology, 200(1):3 LP - 22, 2018. doi: 10.4049/jimmunol.1701494. URL http: //www. jimmunol. org/content/200/1/3. abstract. [p266]

X. Y. Lam, J. Marron, D. Sun, and K.-C. Toh. DWDLargeR: Fast Algorithms for Large Scale Generalized Distance Weighted Discrimination, 2018a. URL https://CRAN. R-project. org/package=DWDLargeR. $\mathrm{R}$ package version 0.1-0. [p268]

X. Y. Lam, J. S. Marron, D. Sun, and K.-C. Toh. Fast Algorithms for Large-Scale Generalized Distance Weighted Discrimination. Journal of Computational and Graphical Statistics, 27(2):368-379, 2018b. ISSN 1061-8600. doi: 10.1080/10618600.2017.1366915. URL https://doi .org/10.1080/10618600. 2017. 1366915. [p268]

E. J. Limkin, R. Sun, L. Dercle, E. I. Zacharaki, C. Robert, S. Reuzé, A. Schernberg, N. Paragios, E. Deutsch, and C. Ferté. Promises and challenges for the implementation of computational medical imaging (radiomics) in oncology. Annals of Oncology, 28(6):1191-1206, 2017. doi: 10.1093/annonc/ mdx034. URL https://doi.org/10.1093/annonc/mdx034. [p266]

J. S. Marron, M. J. Todd, and J. Ahn. Distance-Weighted Discrimination. Journal of the American Statistical Association, 102(480):1267-1271, 2007. doi: 10.1198/016214507000001120. URL https: //doi.org/10.1198/016214507000001120. [p267]

A. E. Nelson, F. Fang, L. Arbeeva, R. J. Cleveland, T. A. Schwartz, L. F. Callahan, J. S. Marron, and R. F. Loeser. A machine learning approach to knee osteoarthritis phenotyping: data from the FNIH Biomarkers Consortium. Osteoarthritis and Cartilage, 27(7):994-1001, 2019. doi: https:// doi.org/10.1016/j.joca.2018.12.027. URL http://www. sciencedirect.com/science/article/pii/ S1063458419309264. [p267]

N. Pinky, S. M. Islam, and R. Alice. Edibility Detection of Mushroom Using Ensemble Methods. International Journal of Image, Graphics and Signal Processing, 11:55-62, 2019. doi: 10.5815/ijigsp.2019. 04.05. [p271]

S. Wei, C. Lee, L. Wichers, and J. S. Marron. Direction-Projection-Permutation for High-Dimensional Hypothesis Tests. Journal of Computational and Graphical Statistics, 25(2):549-569, 2016. doi: 10.1080/ 10618600.2015.1027773. URL https://doi .org/10.1080/10618600.2015.1027773. [p266]

A. Wibowo, Y. Rahayu, A. Riyanto, and T. Hidayatulloh. Classification algorithm for edible mushroom identification. In 2018 International Conference on Information and Communications Technology (ICOIACT), pages 250-253, 2018. doi: 10.1109/ICOIACT.2018.8350746. [p271]

Andrew G. Allmon

University of North Carolina at Chapel Hill

Department of Biostatistics

dallmon@email.unc. edu

J. S. Marron

University of North Carolina at Chapel Hill

Department of Biostatistics

marron@unc.edu

Michael G. Hudgens

University of North Carolina at Chapel Hill

Department of Biostatistics

mhudgens@email.unc.edu 